\title{
Anthropometric Indices and Cardiovascular Risk: Spanish Perspectives
}

\author{
Jesús Millán*, Carlos Recarte, Maria V. Villalba, C. Lopez Gonzalez-Cobos, Maria \\ Gomez Antunez, Blanca Pinilla, Antonio Muiño \\ Department of Internal Medicine, Hospital General Universitario Gregorio Marañón, Dr. Esquerdo, 46, 28007 School of Medicine. Universidad \\ Complutense, Madrid, Spain
}

Received: December 26, 2013; Accepted: May 17, 2014; Published: May 19, 2014

*Corresponding author: Jesus Millán, Hospital General Universitario Gregorio Marañón, Dr. Esquerdo 46, 28007 Madrid, Spain, Tel: 3491 4265169; Fax: 3491 5866728; Email: jesus.millan@madrid.org

\begin{abstract}
In Spain, several studies have examined the importance of cardiovascular risk factors; however, DORICA was the first to analyze the role of each of them individually, thus revealing how much each risk factor contributes to cardiovascular morbidity and mortality.

In terms of weight, $40.12 \%$ of men and $48.87 \%$ of women were classified as normal according to their BMI; $13.2 \%$ of men and $17.5 \%$ of women were classified as obese. As for cholesterol, $57.3 \%$ of men and $53 \%$ of women had levels greater than $200 \mathrm{mg} / \mathrm{dl}, 23 \%$ of men and $17.95 \%$ of women had LDL-cholesterol levels higher than 160 $\mathrm{mg} / \mathrm{dl}$, whereas $15 \%$ of men and $6 \%$ of women had HDL-cholesterol levels below $40 \mathrm{mg} / \mathrm{dl}$. Hypertriglyceridemia (>150 mg/dl) was detected in $28 \%$ of men and $13 \%$ of women. Systolic blood pressure was greater than $140 \mathrm{mmHg}$ in $30 \%$ of men and in $21 \%$ of women; diastolic pressure was higher than $90 \mathrm{mmHg}$ in $23.2 \%$ of men and in $13.6 \%$ of women. Baseline blood sugar levels were above 126 $\mathrm{mg} / \mathrm{dl}$ in $11.2 \%$ of men and in $7.2 \%$ of women aged more than 55 ; the prevalence of diabetes in this age group was $5.3 \%$ and $2.4 \%$, respectively. Smokers accounted for $39 \%$ of the study population, and this proportion was higher in men $(48.1 \%$ of the population compared with $30.2 \%$ women).
\end{abstract}

The prevalence of metabolic syndrome was $10.87 \%$ overall (12.15\% in men and $9.9 \%$ in women). The number of participants with at least 1 major cardiovascular risk factor (arterial hypertension, dyslipidemia, diabetes) was higher in participants with a BMI $>27$ than in those whose BMI was normal. In terms of waist circumference, $25 \%$ of those individuals with risk factors $(>102 \mathrm{~cm}$ in men and $>88$ $\mathrm{cm}$ women) presented at least 1 major cardiovascular risk factor $\left(\chi^{2}=56.970 ; P<0.001\right)$. Receiver operating characteristic (ROC) curves were used to compare the sensitivity and specificity of the different anthropometric indicators and estimate the presence of cardiovascular risk factors associated with obesity.

The individual importance of each cardiovascular risk factor in our setting was evaluated by calculating the attributable risk fraction. The attributable fraction for arterial hypertension was $26.7 \%$ for men and $22.9 \%$ for women. The prevalence of hypercholesterolemia was $20.7 \%$ for men and $18.2 \%$ for women, with an attributable fraction of $15.7 \%$ and $12.7 \%$, respectively. The prevalence of obesity was $13.2 \%$ for men and $17.5 \%$ for women, with an attributable fraction of $4 \%$ and 5\%, respectively. Smoking was third in men, with an attributable fraction of $13.13 \%$, and fourth in women, with an attributable fraction of $3.71 \%$.

\section{Introduction}

Circulatory diseases are the leading cause of mortality in Spain, accounting for $36 \%$ of all deaths. An analysis of mortality patterns in Europe reveals an east-west trend, with the highest rates observed in Eastern Europe. Spain has one of the lowest mortality rates among developed countries [1].

In recent years, the number of deaths due to cerebrovascular disease has fallen considerably, and ischemic heart disease has become the leading cause of cardiovascular death in Spain. However, this number of deaths due to this condition is falling as a result of recent community interventions aimed at reducing risk factors and improving diagnosis, quality of emergency care, and treatment.

Cardiovascular disease is the third cause of years of potential life lost, the leading reason for admission to hospital, and one of the main reasons for disability and failing health.

Coronary ischemic disease is more common in men and mainly affects adults aged 45 to 74 years. Forecasts on cardiovascular disease carried out in Spain reveal that, despite the reduction in mortality rates for cardiovascular disease, the number of persons affected by this condition will increase in the coming years, mainly due to increased longevity of the population and better diagnosis and treatment.

\section{Epidemiology of cardiovascular disease}

Cardiovascular disease is the leading cause of mortality in developed countries, accounting for $36 \%$ of deaths by any cause. The next most common causes of death in our setting are cancer (25\%), respiratory disease (12\%), and digestive disease (5\%).

The most frequent types of cardiovascular disease are ischemic heart disease (31\%) and cerebrovascular disease (29\%). In both, the underlying lesion is atherosclerotic plaque, and cardiovascular risk factors play a major role in its formation. In the category of ischemic heart disease, acute myocardial infarction accounts for $60 \%$ of cases, whereas in cerebrovascular 
disease, cerebral hemorrhage accounts for $19 \%$ of cases, ischemic attack for $16 \%$, and unknown causes for $65 \%$.

In Spain, cardiovascular mortality has recently been falling by approximately $2.4 \%$ annually. This value is mainly the result of the decreased incidence of cerebrovascular disease $3.2 \%$ annually compared with $0.6 \%$ annually for ischemic heart disease). Nevertheless, this decrease is accompanied by an increase in the absolute number of deaths from these causes brought about by aging of the population.

Men account for $55 \%$ of all deaths from cardiovascular disease. However, cardiovascular conditions cause a greater number of deaths among women; therefore, gross cardiovascular mortality is greater for women, both in the general population and in the different age groups, except for the oldest group (>84 years). Therefore, women have a lower cardiovascular risk than men, but greater proportional cardiovascular mortality.

Cardiovascular disease is the leading cause of death overall: the gross cardiovascular mortality rate is $355 / 100,000$ for women and 309/100,000 for men. These figures vary by age group: 1,000/100,000 in individuals aged over 70 years, falling significantly among those aged 40 to 70 years (the second most common cause of death after cancer in this group).

There are considerable differences in cardiovascular mortality in the different Spanish autonomous communities. Those with the greatest incidence are Andalusia, Murcia, Community of Valencia, Balearic Islands and Canary Islands; those with the lowest rates are Madrid, Castile-Leon, Navarre, and La Rioja. This distribution is somewhat paradoxical, as the areas with the highest rates are in the Mediterranean, thus pointing to the possibility of exogenous dietary and socioeconomic factors (some of them unknown) that could be more common in these areas [2].

In comparison with other developed countries, mortality from cardiovascular disease in Spain is considerably lower than in central and northern Europe and the United States. This could be due to the positive influence of the Mediterranean diet on cardiovascular factors.

\section{Cardiovascular Risk Factors}

There is sufficient evidence that independent cardiovascular risk factors-smoking, arterial hypertension, hypercholesterolemia (especially high levels of low-density lipoprotein [LDL] cholesterol), and diabetes mellitus-are common in the population and are the direct cause of ischemic heart disease $[3,4]$.

Classic epidemiology studies show that hypercholesterolemia and arterial hypertension are important risk factors for ischemic heart disease. For cerebrovascular disease, only hypertension has been observed to be a risk factor, whereas hypercholesterolemia has not. Epidemiology studies conclude that the total cholesterol/ high-density lipoprotein (HDL) cholesterol ratio is the best indicator of the effect of lipid metabolism on the risk of coronary disease.

Other cardiovascular risk factors include family history, sedentary lifestyle, socioeconomic level, depression, race, obesity, hypertriglyceridemia, glucose intolerance, elevated levels of homocysteine and apolipoprotein B, or electrocardiographic abnormalities consistent with left ventricular hypertrophy. Hypertriglyceridemia, lipoprotein and homocysteine abnormalities, and some clotting factors are considered conditional risk factors [5]. Others, such as obesity or sedentary lifestyle, are considered predisposing risk factors, as they play a role in the onset of the main or conditional risk factors [6].

Although the relation between obesity and coronary disease is not clear, a positive association has been found between the risk of coronary disease and body mass index (BMI) greater than 18.5. This association is more marked in nonsmokers.

A greater risk has been reported in people with mainly central obesity. Since BMI does not provide information on body fat distribution, different indicators have been proposed for this purpose, such as the waist-to-hip ratio, the waist-to-height ratio, or waist circumference.

The results of the MONICA Study and the Seven Countries Study provide standardized and comparable information on the outcome of risk factors for coronary heart disease in different European countries during the last few decades. Around 1995, the highest rates of cholesterolemia were observed in central Europe and the lowest rates in southern Europe, specifically in Catalonia. There were no differences in blood pressure between the north and the south.

Studies analyzing the dose-response effect between cardiovascular risk factors and ischemic heart disease have revealed that the impact of arterial hypertension and hypercholesterolemia is gradual both in northern Europe and in southern Europe with respect to Mediterranean countries for cholesterol levels of $200 \mathrm{mg} / \mathrm{dL}$. The absolute risk was 3 times greater in northern Europe for blood pressure levels of 140 $\mathrm{mmHg}$.

The MONICA study revealed that blood cholesterol levels and blood pressure have decreased during the last decade, although during this time, there has been a considerable increase in mean BMI and in the prevalence of obesity.

As for risk factors that can be controlled, including arterial hypertension and smoking, comparative cost-effectiveness analyses carried out to date indicate that strategies aimed at healthy eating habits are more important than strategies based on drugs to reduce blood cholesterol levels $[7,8]$.

\section{Prognostic factors for cardiovascular and atherosclerotic disease}

As it is well know the main factors associated with the development and outcome of cardiovascular disease are as follows:

\section{Risk factors for cardiovascular disease}

\section{Parameters used to stratify risk:}

- Systolic and diastolic blood pressure (Grade 1-3) 
- Men aged $>55$ years

- Women aged $>65$ years

- Smoking

- Total cholesterol > $6.5 \mathrm{mmol} / \mathrm{L}(250 \mathrm{mg} / \mathrm{dL})$.

- Diabetes

- Family history of premature cardiovascular disease

Other factors that negatively affect prognosis:

- Low HDL cholesterol

- High LDL cholesterol

- Microalbuminuria in diabetes

- Carbohydrate intolerance

- Obesity

- Sedentary lifestyle

- High fibrinogen

- High-risk socioeconomic group

- High-risk ethnic group

- High-risk geographic region

\section{Target organ lesion:}

- Left ventricular hypertrophy diagnosed by electrocardiogram or echocardiography

- Proteinuria, mild increase in plasma creatinine concentration (1.2-2 mg/dL), or both.

- Echographic or radiologic signs of the presence of atherosclerotic plaque in the carotid, iliac, or femoral arteries, or in the aorta.

- Focal or generalized stenosis of retinal arteries.

\section{Associated clinical disorders:}

- Cerebrovascular disease: Cerebrovascular accident, cerebral hemorrhage, or transient ischemic attack.

- Heart disease: Myocardial infarction, angina, coronary revascularization, or congestive heart failure.

- Kidney disease: Diabetic kidney disease or kidney failure (plasma creatinine concentration $>2 \mathrm{mg} / \mathrm{dL}$ ).

- Vascular disease: Dissecting aneurysm or symptomatic artery disease.

- Advanced hypertensive retinal disease: Hemorrhage, exudates, or papillary edema.

\section{The problem to calculate cardiovascular global and} individual risk

Tables based on data from the two main studies on overall cardiovascular risk-Framingham and Score-can be used to calculate cardiovascular risk. The different models for this calculation provide specific information on the risk of having a coronary or cerebrovascular episode during the next five years.

The importance of establishing cardiovascular risk lies in awareness of the need for intervention depending on the level of risk and implementation of an intervention. As the intervention should be as cost-effective as possible, it is important to prioritize therapeutic decisions in patients classed as having a high cardiovascular risk [9].

The most widely used calculation models express risk as the probability (percentage) of experiencing a cardiovascular episode in the next 10 years. Risk is classified as follows:

a. High risk: patients with clinically demonstrated atherosclerotic disease, patients with a calculated risk greater than $20 \%$, and patients with type 2 diabetes.

b. Moderate risk: patients with risk factors and a cardiovascular risk calculated to be between $10 \%$ and $20 \%$.

c. Low risk: patients with a cardiovascular risk calculated to be below $10 \%$.

Spanish perspective from recent studies (Tables 1, 2, 3 and 4)

The DORICA (Dislipemia, Obesidad y RIesgo Cardiovascular [Dyslipidemia, Obesity, and Cardiovascular Risk]) Study [10] was carried out by a Spanish working group analyzing obesity and cardiovascular risk. One of the reasons this expert panel was established was the need for reliable data from the Spanish population in terms of the prevalence of different cardiovascular risk factors and their association with eating habits and with anthropometric data in terms of obesity. So, the interest was focused:

- To ascertain the prevalence of cardiovascular risk factors in the Spanish population.

- To analyze the impact of obesity on cardiovascular risk factors.

- To propose a quantitative or qualitative model for estimation of cardiovascular risk factors in our setting using other models.

- To calculate the fraction of attributable risk for each of the risk factors analyzed with respect to the Spanish population.

Data of the study were collected from epidemiology studies on nutrition and risk factors using random samples that were representative of the populations of the autonomous regions of Andalusia, Balearic Islands, Canary Islands, Catalonia, Galicia, Madrid, Murcia, Basque Country, and Valencia during the period 1990 and 2000. A multistage random sampling procedure was followed in all cases with stratification by age, gender, and residence, in proportion with population density. Data were collected by qualified health care staff that was trained according to the requirements of the study protocol. 
Table 1: Distribution of Anthropometric Parameters and systolic/Diastolic blood pressure by age.

\begin{tabular}{|c|c|c|c|c|c|c|c|}
\hline AGE GROUP & LIPID PARAMETER & MEAN & SD & $95 \% \mathrm{CI}$ & 25th & $\begin{array}{c}\text { 50th } \\
\text { Percentiles }\end{array}$ & 75th \\
\hline \multirow[t]{7}{*}{$25-34$} & Weight (Kg) & $75,11^{\mathrm{a}}$ & 10,89 & $74,63-75,59$ & 67,20 & 74,00 & 82,00 \\
\hline & Height (cm) & $173,51^{\mathrm{a}}$ & 676 & $173,22173,81$ & 169 & 173,00 & 178,00 \\
\hline & BMI (kg/m2) & $24,91^{\mathrm{a}}$ & 3,27 & $24,76-25,05$ & 22,75 & 24,54 & 26,85 \\
\hline & Waist (cm) & $87,15^{\mathrm{a}}$ & 8,66 & $86,62-87,68$ & 80,50 & 86,50 & 93,00 \\
\hline & Waist/Hip ratio & $0,89^{\mathrm{a}}$ & 0,06 & $0,89-0,89$ & 0,85 & 0,89 & 0,93 \\
\hline & SBP (mmHg) & $126,24^{\mathrm{a}}$ & 13,58 & $125,49126,98$ & 118,00 & 125,00 & 135,00 \\
\hline & DBP(mmHg)50th & $76,98^{a}$ & 10,07 & 76,43-7753 & 70,00 & 76,00 & 84,00 \\
\hline \multirow[t]{7}{*}{$35-44$} & Weight (Kg) & 76,26 & 11,40 & $75,72-76,80$ & 69,00 & 75,00 & 83,00 \\
\hline & Height (cm) & 171,09 & 6,63 & $170,77171,40$ & 166,87 & 171,00 & 175,50 \\
\hline & BMI (kg/m2) & 26,01 & 3,41 & $25,85-26,17$ & 23,78 & 25,83 & 28,01 \\
\hline & Waist (cm) & 91,16 & 9,78 & $90,52-91,80$ & 84,00 & 91,50 & 96,50 \\
\hline & Waist/Hip ratio & 0,91 & 0,06 & $0,91-0,92$ & 0,88 & 0,91 & 0,95 \\
\hline & SBP (mmHg) & 127,85 & 14,85 & $126,97128,72$ & 120,00 & 127,50 & 137,00 \\
\hline & DBP (mmHg) & 79,85 & 11,37 & 79,18-80,51 & 71,00 & 80,00 & 88,00 \\
\hline \multirow[t]{7}{*}{$45-54$} & Weight (Kg) & 76,38 & 11,58 & 75,78-76,98 & 69,00 & 75,00 & 83,00 \\
\hline & Height (cm) & 169,00 & 7,09 & $168,63169,37$ & 164,00 & 169,00 & 173,91 \\
\hline & BMI (kg/m2) & 26,71 & 3,62 & $26,52-26,89$ & 24,52 & 26,46 & 28,86 \\
\hline & Waist (cm) & 94,46 & 9,72 & $93,77-95,15$ & 88,40 & 94,00 & 100,00 \\
\hline & Waist/Hip ratio & 0,94 & 0,06 & $0,94-0,95$ & 0,90 & 0,94 & 0,99 \\
\hline & SBP (mmHg) & 133,11 & 15,35 & $132,11134,12$ & 120,00 & 130,00 & 144,50 \\
\hline & DBP (mmHg) & 83,11 & 12,05 & $82,32-83,90$ & 75,00 & 83,50 & 90,00 \\
\hline \multirow[t]{7}{*}{$55-64$} & Weight (Kg) & 75,82 & 10,82 & $75,20-76,45$ & 69,10 & 75,50 & 82,00 \\
\hline & Height (cm) & 167,15 & 6,86 & $166,76167,55$ & 163,00 & 167,50 & 172,00 \\
\hline & BMI (kg/m2) & 27,10 & 3,48 & $26,90-27,30$ & 24,92 & 26,57 & 29,34 \\
\hline & Waist (cm) & 96,85 & 8,93 & $96,14-97,56$ & 91,50 & 97,00 & 102,30 \\
\hline & Waist/Hip ratio & 0,96 & 0,06 & $0,96-0,96$ & 0,92 & 0,97 & 1,00 \\
\hline & SBP (mmHg) & 139,44 & 18,20 & $138,11-140,77$ & 128,00 & 139,50 & 150,00 \\
\hline & DBP (mmHg) & 84,62 & 11,01 & $83,82-85,42$ & 78,00 & 85,00 & 90,00 \\
\hline
\end{tabular}

Table 2: Lipid Profile by Age Group.

\begin{tabular}{|l|l|c|c|c|c|c|c|}
\hline AGE GROUP & LIPID PARAMETER & MEAN & SD & $\mathbf{9 5 \%}$ CI & 25th & $\begin{array}{c}\mathbf{5 0 t h} \\
\text { Percentiles }\end{array}$ & $\mathbf{7 5 t h}$ \\
\hline $\mathbf{2 5 - 3 4}$ & Total cholesterol (mg/dL) & $192,97^{\text {a }}$ & 35,77 & $188,80-197,13$ & 169,00 & 191,00 & 216,94 \\
\hline & cLDL (mg/dL) & $121,20^{\text {a }}$ & 32,99 & $116,86-125,53$ & 97,00 & 119,94 & 143,00 \\
\hline & cHDL (mg/dL) & 52,15 & 12,28 & $50,71-53,48$ & 44,09 & 51,00 & 58,00 \\
\hline & Triglycerides (mg/dL) & $119,14^{\text {b }}$ & 69,10 & $111,09-127,18$ & 76,86 & 107,28 & 144,75 \\
\hline & Glucemia (mg/dL) & 91,81 & 11,00 & $90,34-93,28$ & 85,06 & 92,00 & 98,00 \\
\hline & Total cholesterol(mg/dL) & 211,8 & 43,31 & $206,75-217,21$ & 181,59 & 212,96 & 238,95 \\
\hline & cLDL (mg/dL) & 136,2 & 38,03 & $131,48-141,96$ & 109,00 & 137,00 & 159,23 \\
\hline & cHDL (mg/dL) & 51,76 & 14,03 & $50,05-53,46$ & 43,00 & 49,21 & 61,00 \\
\hline & Triglycerides (mg/dL) & 142,78 & 130,67 & $126,98-158,58$ & 78,12 & 120,00 & 158,08 \\
\hline & Glucemia (mg/dL) & 96,75 & 21,28 & $93,77-99.73$ & 86,84 & 94,00 & 102,00 \\
\hline & Total cholesterol (mg/dL) & 215,54 & 36,52 & $212,12-222,96$ & 196,00 & 221,00 & 241,00 \\
\hline & cLDL (mg/dL) & 143,26 & 36,38 & $138,03-148,49$ & 118,00 & 145,02 & 162,48 \\
\hline
\end{tabular}




\begin{tabular}{|l|l|c|c|c|c|c|c|}
\hline & cHDL (mg/dL) & 50,40 & 12,99 & $48,71-52,09$ & 42,00 & 48,00 & 57,00 \\
\hline & Triglycerides (mg/dL) & 132,18 & 74,85 & $122,46-141,89$ & 77,00 & 116,82 & 168,11 \\
\hline & Glucemia (mg/dL) & 101,41 & 26,11 & $97.53-105.29$ & 90,00 & 97,86 & 105,00 \\
\hline & Total cholesterol (mg/dL) & 217,54 & 36,52 & $212,12-222,96$ & 196,00 & 221,00 & 241,00 \\
\hline & cLDL (mg/dL) & 141,96 & 31,08 & $136,76-147,16$ & 125,00 & 147,80 & 163,00 \\
\hline & cHDL (mg/dL) & 52,36 & 16,08 & $49,96-54,75$ & 42,02 & 50,00 & 60,99 \\
\hline & Triglycerides (mg/dL) & 141,41 & 86,04 & $128,62-154,20$ & 94,33 & 118,58 & 165,29 \\
\hline & Glucemia (mg/dL) & 104,11 & 27,67 & $99,56-108,66$ & 89,00 & 97,00 & 107,00 \\
\hline
\end{tabular}

Table 3: Prevalence of Cardiovascular risk factors in the Spanish population.

\begin{tabular}{|c|c|c|c|c|}
\hline \multirow[b]{2}{*}{ RISK FACTOR } & \multicolumn{2}{|c|}{ MEN } & \multicolumn{2}{|l|}{ WOMEN } \\
\hline & $\begin{array}{l}\text { Prevalence } \\
(95 \% \mathrm{CI})\end{array}$ & $\begin{array}{c}\text { Attributable fraction } \\
(\%)\end{array}$ & $\begin{array}{l}\text { Prevalence } \\
\text { (95\%CI) }\end{array}$ & Attributable fraction (\%) \\
\hline \multicolumn{5}{|c|}{ Hypercholesterolemia } \\
\hline$>200 \mathrm{mg} / \mathrm{dL}$ & $57,3 \%(56,96-57,64)$ & 34 & $53 \%(52,68-53,32)$ & 30 \\
\hline$>240 \mathrm{mg} / \mathrm{dL}$ & $20,7 \%(52,68-53,32)$ & 16 & $18,21 \%(17,99-18,43)$ & 13 \\
\hline \multicolumn{5}{|l|}{ Hypertension } \\
\hline$>140 / 90 \mathrm{mmHg}$ & $36,4 \%(35.55-37.24)$ & 27 & $24.8 \%(24,36-25,24)$ & 23 \\
\hline Diabetes & $5,3 \%(4,92-5,68)$ & 6 & $2,4 \%(2,03-2,77)$ & 3 \\
\hline Smoking & $48,1 \%(47,57-48,63)$ & 13,1 & $30,2 \%(29,64-30,76)$ & 4 \\
\hline Obesity & $13,2 \%(13,09-13,31)$ & 4 & $17,5 \%(17,28-17,72)$ & 5 \\
\hline
\end{tabular}

Table 4: Sociodemographic Characteristics.

\begin{tabular}{|c|c|c|}
\hline AGE GROUP & MEN & WOMEN \\
\hline $25-34$ & 35,0 & 32,5 \\
\hline $35-44$ & 27,5 & 28,4 \\
\hline $45-54$ & 21,3 & 23,9 \\
\hline $55-64$ & 16,2 & 15,3 \\
\hline \multicolumn{3}{|l|}{ EDUCATIONAL LEVEL } \\
\hline LOW & 23,17 & 28,46 \\
\hline MEDIUM & 40,81 & 43,85 \\
\hline HIGH & 36,02 & 27,69 \\
\hline \multicolumn{3}{|l|}{ SOCIOECONOMIC LEVEL } \\
\hline LOW & 22,96 & 27,25 \\
\hline MEDIUM & 66,03 & 66,08 \\
\hline HIGH & 11,02 & 6,67 \\
\hline \multicolumn{3}{|l|}{ AREA OF RESIDENCE } \\
\hline$<10.000$ inhabitants & 12,09 & 11,81 \\
\hline $10.000-100.000$ inhabitants & 31,81 & 31,98 \\
\hline$>100.000$ inhabitants & 56,11 & 56,14 \\
\hline \multicolumn{3}{|l|}{ REGION } \\
\hline North & 11,73 & 11,66 \\
\hline Northwest & 6,78 & 6,89 \\
\hline Center & 25,98 & 26,14 \\
\hline Northeast & 18,80 & 18,68 \\
\hline East & 14,64 & 14,67 \\
\hline South & 22,03 & 21,93 \\
\hline
\end{tabular}


The study included the healthy adult non institutionalized population between the ages of 25 and 64 years $(n=14,616$; 6796 men and 7820 women). The sample was stratified by autonomous region, age group, and gender, and was adjusted and weighted for data analysis in accordance with the intercensal population estimate for Spain in 1998.

An analysis of variables must be included:

- $\quad$ Socio-demographic

1. Age group: 25-34 years, 35-44 years, 45-54 years, 55-64 years.

2. Gender: man, woman.

3. Marital status: single, married, divorced-separated, widowed.

4. Educational level: low, medium, high.

5. Social class: low, middle, high.

6. Area of residence: $<10,000$ inhabitants, $10,000-100,000$ inhabitants, $>100,000$ inhabitants.

7. Region.

- $\quad$ Lifestyle

1. Eating habits: The analysis of eating habits was made using the 24-hour recall method ( 2 or 3 nonconsecutive days) or diet diaries on 3 nonconsecutive days [11].

2. Smoking: smoker, ex-smoker, nonsmoker.

3. Physical activity: very low, low, medium, high, very high.

4. Exercise (sport): yes, no.

5. Frequency of exercise (sport).

6. Alcohol: yes, no.

7. Sleep.

- $\quad$ Anthropometry

1. Weight

2. Height

3. BMI

4. Waist and hip circumferences

5. Systolic and diastolic blood pressure: in the dominant arm with two separate sequential measurements. Blood pressure was considered the mean of the two readings. Arterial hypertension was considered as systolic pressure $>140 \mathrm{mmHg}$ diastolic pressure $>90 \mathrm{mmHg}$, or both.

6. Waist-to-hip ratio

7. Waist-to-height ratio

8. Obesity: calculated as $\mathrm{BMI}>30$

- $\quad$ Biochemistry

1. Baseline blood sugar
2. Total cholesterol: $<200 \mathrm{mg} / \mathrm{dl}$ (desirable), $200-239 \mathrm{mg} /$ dl, $>240 \mathrm{mg} / \mathrm{dl}$ (high).

3. LDL cholesterol: $<100 \mathrm{mg} / \mathrm{dl}, \quad 100-129 \mathrm{mg} / \mathrm{dl}$ (desirable), $130-159 \mathrm{mg} / \mathrm{dl}, 160-189 \mathrm{mg} / \mathrm{dl}$ (high), $>190 \mathrm{mg} / \mathrm{dl}$.

4. HDL cholesterol: $<40 \mathrm{mg} / \mathrm{dl}$ (low), $40-59 \mathrm{mg} / \mathrm{dl}$ (normal), $>60 \mathrm{mg} / \mathrm{dl}$.

5. Triglycerides: $<150 \mathrm{mg} / \mathrm{dl}, \mathbf{1 5 0 - 1 9 9} \mathrm{mg} / \mathrm{dl}$ (high), 200$499 \mathrm{mg} / \mathrm{dl},>500 \mathrm{mg} / \mathrm{dl}$.

- $\quad$ Other

Metabolic syndrome: defined as a fulfilling 3 or more of the following:

- Abdominal obesity: waist circumference $>102 \mathrm{~cm}$ for men or $>88 \mathrm{~cm}$ for women

- Blood pressure >130/85 mmHg.

- Baseline blood sugar $>110 \mathrm{mg} / \mathrm{dl}$.

- Hypertriglyceridemia $>150 \mathrm{mg} / \mathrm{dl}$.

- HDL-cholesterol $<40 \mathrm{mg} / \mathrm{dl}$ for men or $<50 \mathrm{mg} / \mathrm{dl}$ for women

- Measurement of anthropometric variables

- Weight was determined in kilograms with a tolerance of $\pm 100 \mathrm{~g}$. Height was measured in centimeters with a tolerance of $\pm 0.5 \mathrm{~cm}$.

- The BMI was used for the ponderal index. This divides body weight in kilograms by the square of height in meters (body weight $[\mathrm{kg}] /$ height $\left[\mathrm{m}^{2}\right]$ ). Participants were classified as normal weight if the BMI was between 18.5 and 24.9, overweight grade I if the BMI was between 25 and 26.9, overweight grade II if the BMI was between 27 and 29.9, obese type I if BMI was between 30 and 34.9, obese type II if BMI was between 35 and 39.9, obese type III or morbidly obese if BMI was above 40 , and obese type IV or extremely obese if the BMI was above 50 .

Methodological considerations including as follows:

- The statistical analysis was performed using SPSS and Stata. Proportions were estimated using 95\% confidence intervals.

- Quantitative variables were expressed as the mean and standard deviation (SD). Statistical significance was set at $P$ $<0.05$.

- Analysis of variance was performed to compare the mean between groups; the $\chi^{2}$ was performed to compare proportions.

- The fraction of risk attributable to each factor was calculated to evaluate the individual importance of each factor in our setting.

\section{A critical analysis to action}

In terms of weight, $40.12 \%$ of men and $48.87 \%$ of women were classified as normal according to their BMI; $13.2 \%$ of men and $17.5 \%$ of women were classed as obese. Prevalence increased with age and was significantly higher in women aged more than 45 years [12]. 
As for cholesterol, $57.3 \%$ of men and $53 \%$ of women had levels greater than $200 \mathrm{mg} / \mathrm{dl}, 23 \%$ of men and $17.95 \%$ of women had LDL-cholesterol levels higher than $160 \mathrm{mg} / \mathrm{dl}$, whereas $15 \%$ of men and $6 \%$ of women had HDL-cholesterol levels below 40 $\mathrm{mg} / \mathrm{dl}$. Hypertriglyceridemia (>150 mg/dl) was detected in $28 \%$ of men and $13 \%$ of women [13]

Systolic blood pressure was greater than $140 \mathrm{mmHg}$ in $30 \%$ of men and in $21 \%$ of women; diastolic pressure was higher than 90 $\mathrm{mmHg}$ in $23.2 \%$ of men and in $13.6 \%$ of women. The prevalence of arterial hypertension increased with age in both sexes, with a greater prevalence in men up to 54 years, after which age it leveled off.

Baseline blood sugar levels were above $126 \mathrm{mg} / \mathrm{dl}$ in $11.2 \%$ of men and in $7.2 \%$ of women aged more than 55 ; the prevalence of diabetes in this age group was $5.3 \%$ and $2.4 \%$, respectively.

Smokers accounted for 39\% of the study population, and this proportion was higher in men $(48.1 \%$ of the population compared with $30.2 \%$ women).

Significantly higher values were observed in BMI and hip circumference as arterial hypertension worsened; thus, participants classed as grade II-IV had a mean BMI value of 27.2 and a hip circumference of $94.5 \mathrm{~cm}$. Similarly, mean values for total cholesterol, LDL-cholesterol, and triglycerides increased significantly with BMI in both men and women.

The prevalence of metabolic syndrome was $10.87 \%$ overall (12.15\% in men and $9.9 \%$ in women). The number of participants with at least 1 major cardiovascular risk factor (arterial hypertension, dyslipidemia, diabetes) was higher in participants with a BMI $>27$ than in those whose BMI was normal. In terms of waist circumference, $25 \%$ of those individuals with risk factors ( $>102 \mathrm{~cm}$ in men and $>88 \mathrm{~cm}$ women) presented at least 1 major cardiovascular risk factor $\left(\chi^{2}=56.970 ; P<0.001\right)$.

Receiver operating characteristic (ROC) curves were used to compare the sensitivity and specificity of the different anthropometric indicators and estimate the presence of cardiovascular risk factors associated with obesity. The conclusion was that waist circumference, with an area under the curve of 0.7499 in men and 0.7080 in women, was the variable with the most significant results, followed by waist-to-hip ratio (0.7502 and 0.7163 , respectively).

The individual importance of each cardiovascular risk factor in our setting was evaluated by calculating the attributable risk fraction, using data from the Framingham study as a comparison as follows: PAR $=[\mathrm{P}(\mathrm{RR}-1) /(1+\mathrm{P}(\mathrm{RR}-1)] \times 100$, where PAR is the population-attributable risk, $\mathrm{P}$ the prevalence of the risk factor, and RR the relative risk estimated for the risk factor in the Framingham study.

The prevalence of arterial hypertension was $36.4 \%$ in men $24.8 \%$ in women; therefore, the attributable fraction for arterial hypertension was $26.7 \%$ for men and $22.9 \%$ for women. The prevalence of hypercholesterolemia was $20.7 \%$ for men and $18.2 \%$ for women, with an attributable fraction of $15.7 \%$ and
$12.7 \%$, respectively. The prevalence of obesity was $13.2 \%$ for men and $17.5 \%$ for women, with an attributable fraction of $4 \%$ and $5 \%$, respectively. Smoking was third in men, with an attributable fraction of $13.13 \%$, and fourth in women, with an attributable fraction of $3.71 \%$.

\section{Towards the future}

The recent studies showed that the greater the number of risk factors, the greater the risk of suffering from a cardiovascular disease, although specificity and sensitivity for each of them independently were low.

Although the population samples used in the DORICA study were cross-sectional and from different studies, we observed that, after adjustment and weighting of sample data, estimations can be made on a representative spectrum of the Spanish population from different regions. Therefore, the data collected in each of the autonomous communities can be considered as representative.

The main limitations of different studies lie in the absence of data on the presence of coronary disease or other specific cardiovascular conditions. Given that most studies in Spain are also affected by this limitation, data on mortality and cardiovascular disease are being collected in the different autonomous communities, as is the case with the IBERICA study.

Other research projects carried out recently in Spain on the importance of cardiovascular risk factors are MONICA and the National Health Survey, which, despite providing a general vision of the prevalence of cardiovascular disease in Spain, lack the individual anthropometric and biochemistry data necessary to draw a greater number of conclusions.

Some studies have also analyzed the quality of the information in clinical histories in primary care with respect to cardiovascular risk factors. They conclude that there has been a notable improvement in recent years, except in the frequency of recording information on smoking and alcohol.

The DORICA study stresses the need to continue developing programs based on dietary habits, physical exercise, and early detection of risk factors as indispensable approaches to preventing coronary disease $[14,15]$.

\section{Priority actions points for the prevention of cardiovascular disease in Spain}

The results of recent studies, together with clinical and epidemiological evidence from previous studies, point to a number of areas that can help prevent cardiovascular disease in Spain.

I. Lifestyle: Insistence by primary care physicians on smoking cessation, a healthy diet, and a minimum of daily physical exercise.

II. Smoking cessation programs: Informing the public of the many adverse effects of smoking on health, completely suppressing direct or indirect advertising that promotes consumption, and ensuring compliance with the law preventing smoking in public places. 
III. Healthy diet: Dietary recommendations: consumption of fruit, vegetables, pulses, and cereals; reduction of fatty foods, especially those containing saturated fats, refined sugar, and salt; minimum intake of omega 3 , oleic acid, vitamin $B$, and antioxidants.

Support for companies from the food industry that promote strategies to provide healthy food at lower prices. Control of food advertising and support for campaigns that promote healthy foods.

IV. Physical activity: Promotion of individual and community exercise plans adapted to individual needs and characteristics. Recommendation of a medical check-up and a design of a progressive training program before starting a training program.

V. Early detection and treatment of the main cardiovascular risk factors: Hypercholesterolemia, arterial hypertension, smoking, carbohydrate intolerance, diabetes mellitus, overweight, obesity, and metabolic syndrome.

VI. Assessment of cardiovascular risk on an individual basis: Initiation of strategies that encourage healthy lifestyles in patients with a moderate or high risk. Starting treatment with drugs if a healthy lifestyle does not prove effective at controlling risk factors permanently.

VII. Control of atherosclerotic disease: In patients with signs of ischemic heart disease, peripheral artery disease, or cerebrovascular disease. Cardiovascular risk factors should be treated early, and specific treatment of the cardiovascular disease should be started.

VIII. Avoid alcohol: Never more than two low-proof drinks per day for a man, and half this amount for a woman.

IX. Support from government and the media for health programs: Promoting health in schools and the workplace, with social support for health programs.

X. Promote research on cardiovascular disease: Increase resources for promoting research for scientific societies, so that better results are generated and more reliable therapy designed.

\section{Summary Points}

1. Cardiovascular disease is the main cause of death in developed countries. Spain is one of the countries with a lower mortality index.

2. Although the cardiovascular mortality rate is falling in Spain, the total number of deaths will increase due to aging of the population.

3. The importance of the DORICA study lies in the fact that it is the first in Spain to investigate the attributable fraction of each cardiovascular risk factor individually.

4. Cardiovascular risk should be calculated on an individual basis to provide tailored strategies and take full advantage of these strategies.

5. The most important anthropometric values for subsequent cardiovascular risk were waist diameter and waist-to-height index.

6. Although the total number of deaths from cardiovascular disease is greater in men, the gross mortality rate is higher in women.

7. Obesity is a risk factor with little impact on cardiovascular mortality, although it is significantly associated with other key risk factors, such as arterial hypertension and hyperglycemia.

8. Early detection of risk factors and promotion of a healthy diet and lifestyle are particularly important as cardiovascular risk reduction strategies.

9. Future studies in Spain must determine the association between risk factors and the number of cases affected by coronary disease and cerebrovascular disease, since we currently lack protocols of this type.

\section{References}

1. Regidor E, Gutierrez-Fisac JL, Calle ME, Otero A (2002) Patron de mortalidad en Espana, 1998. Med Clin (Barc) 118(1): 13-15.

2. Tormo Diaz MJ, Navarro MC, Chirlaque MD, Perez FD (1997) Factores de riesgo cardiovascular en la Region de Murcia, Espana. Rev Esp Salud Publica 71(6): 515-529.

3. Gaede P, Vedel P, Larsen N, Jensen GV, Parving HH, et al. (2003) Multifactorial intervention and cardiovascular disease in patients with type 2 diabetes. N Engl J Med 348(5): 383-393.

4. Murray CJ, Lopez AD (1997) Mortality by cause for eight regions of the World: Global burden of disease study. Lancet 349(9061): 1269-1276.

5. Eckel RH (1997) Obesity and heart disease. Circulation 96: 3248-3250.

6. Sociedad Espanola para el Estudio de la Obesidad (SEEDO) (1996) Consenso espanol para la evaluacion de la obesidad y para la realizacion de estudios epidemiologicos. Med Clin (Barc) 107: 782787.

7. Maiques A, Brotons C, Villar F (1997) Guias de prevencion cardiovascular. PAPPS-Semfyc, Barcelona.

8. World Health Organization (WHO) (2003) Diet, nutrition and the prevention of chronic diseases: report of a joint FAO/WHO Expert consultation. WHO Technical report series 916, Geneva.

9. Sociedad Espanola para el Estudio de la Obesidad (SEEDO) (2007) Consenso SEEDO 2007 para la evaluacion del sobrepeso y la obesidad y el establecimiento de criterios de intervencion terapeutica. Med Clin (Barc)

10. Aranceta J, Foz M, Gil B, Jover E, Mantilla T, et al. (2004) Obesidad y Riesgo Cardiovascular. Estudio Dorica 15(5): 196-233.

11. Aranceta J, Perez C, Amela C, Garcia Herrera R (1994) Encuesta de Nutricion de la Comunidad de Madrid. Madrid: Consejeria de Salud de la Comunidad de Madrid.

12. Aranceta J, Perez-Rodrigo C, Serra Majem Ll, Ribas Barba L, Quiles Izquierdo J et al. (2003) y el Grupo Colaborativo para el estudio de la Obesidad en Espana, Med Clin (Barc) 120: 608-612.

13. Rubies-Prat J, Botet JP (2003) Hypertrigliceridemia como factor de riesgo cardiovascular. ¿Fin de la controversia?. Med Clin (Barc) 120(8): 303-307.

14. NIH. National Institutes of Health National Heart, Lung and Blood 
Institute. Clinical guidelines on the identification, evaluation and treatment of overweight and obesity in adults. The evidence report. Bethesda (MD, USA).
15. Renaud S, Lanzmann-Petithory D (2001) Coronary heart disease: dietary links and pathogenesis. Public Health Nutr 4(2B): 459-474. 